\title{
SWEET’S SYNDROME AND RHEUMATOID ARTHIRTIS: A CASE REPORT
}

\section{Thalita do Nascimento Silva ${ }^{1, \star}$, Francisco Helialdo Sousa de Oliveira Filho ${ }^{1}$, Priscila Dourado Evangelista ${ }^{1}$, Rodrigo Barbosa} de Azevedo ${ }^{1}$, Déborah Pereira Gonçalves ${ }^{1}$

1.Hospital Geral Dr. César Cals, Fortaleza (CE), Brazil.

*Corresponding author: thalitadns@gmail.com

\section{BACKGROUND}

Sweet's syndrome (SS), an acute febrile neutrophilic dermatosis, is a rare illness, characterized by painful skin lesions like plaques, nodules and papules, fever, leukocytosis with neutrophilia and histopathological finding of neutrophilic inflammatory infiltrate in the dermis. This disease can be associated with malignancy, autoimmune diseases, infections or drugs and has a dramatic response to corticosteroids.

\section{CASE REPORT}

A 58-year-old woman was diagnosed with rheumatoid arthritis (RA) 11 years ago with register of rheumatoid factor (RF) and anticyclic citrullinated peptide (anti-CCP) at high titers, in use of tocilizumab. On January 2020, the patient had acute skin lesions (papules and nodules) in face, abdomen and limbs, in addition to fever $39^{\circ} \mathrm{C}$, arthralgias and arthritis in hands, wrists, knees and ankles. Tocilizumab was suspended (the last infusion administered on December 2019). Laboratory evaluation showed high levels of erythrocyte sedimentation rate (ESR) and C-reactive protein (CRP), while the blood culture and urine culture had no bacterial growth. In addition, no leukocytosis with neutrophilia was recorded on the complete blood count. Patient denied symptoms compatible with upper respiratory or gastrointestinal infections. Skin biopsy showed neutrophilic dermatosis. The raster for malignancy (lungs, thyroid, stomach, colon, breast, endometrium and cervix) has no neoplasm. The diagnosis of SS is defined by the 1986 Su and Liu's criteria, modified by von der Driesch in 1994, in which the major criteria are: abrupt onset of erythematous and painful nodules or plaques and histopathological evidence of neutrophilic infiltrate in the dermis without leukocytoclastic vasculitis; while minor criteria are: skin lesion preceded by fever or infection, skin lesions accompanied by fever, arthralgia, conjunctive or neoplasm, leukocytosis, elevation of ESR and CRP, good response to corticosteroids. For the diagnosis of SS, two major and at least two minor criteria are required.

\section{CONCLUSION}

The patient presented sufficient criteria for the diagnosis of SS (two major criteria and three minor criteria), which in this situation may be secondary to RA. Treatment with prednisolone $0.5 \mathrm{mg} / \mathrm{kg} /$ day was started. The patient in the case had improve skin lesions and had no new episodes of fever after the use of glucocorticoids. 